\title{
Optimal Low-Complexity Detection for Space Division Multiple Access Wireless Systems
}

\author{
Yue Wu, Tao Cui, and Chintha Tellambura, Senior Member, IEEE
}

\begin{abstract}
A symbol detector for wireless systems using space division multiple access (SDMA) and orthogonal frequency division multiplexing (OFDM) is derived. The detector uses a sphere decoder (SD) and has much less computational complexity than the naive maximum likelihood (ML) detector. We also show how to detect non-constant modulus signals with constrained least squares (CLS) receiver, which is designed for constant modulus (unitary) signals. The new detector outperforms existing suboptimal detectors for both uncoded and coded systems.
\end{abstract}

Index Terms-OFDM, SDMA.

\section{INTRODUCTION}

S PACE division multiple access (SDMA) and Orthogonal frequency division multiplexing (OFDM) can be combined to exploit the advantages of both [1], [2]. In the uplink channel, the base station handles SDMA while the user terminals use OFDM. This asymmetry keeps the overall cost low. Minimum mean squared error (MMSE) detectors [1] perform worse than the ML detector. The detector [2] is designed specifically for unitary signals and outperforms the MMSE detector. A minimum bit error rate multiuser detector is designed in [3]. Reference [4] develops two linear demodulators for minimizing the probability of error. A multistage sensitive bits detector is developed in [5]. However, some of these detectors perform significantly worse than the ML detector.

In this letter, we propose a new detector based on a sphere decoder (SD) for SDMA/OFDM wireless systems. The SD [6] has an average complexity that is roughly polynomial in the number of symbols for a wide range of signal-to-noise ratios (SNRs) [7]. Thus our optimal detector for SDMA/OFDM has much less computational complexity than the naive ML detector. We also show how the CLS detector of [2] can be modified to detect non-unitary signals.

Notation: $(\cdot)^{H}$ and $(\cdot)^{\dagger}$ denote conjugate transpose and Moore-Penrose pseudo-inverse, respectively.

\section{SDMA/OFDM SYSTEM MODEL}

We consider the uplink of an SDMA/OFDM system (Fig. 1) with $U$ users and a basestation (BS). Each user transmits with a single antenna and the $\mathrm{BS}$ has $A \geq 1$ receive antennas.

Manuscript received August 25, 2005. The associate editor coordinating the review of this letter and approving it for publication was Dr. Murat Uysal. This work has been supported in part by the Natural Sciences and Engineering Research Council of Canada, Informatics Circle of Research Excellence and Alberta Ingenuity Fund.

Y. Wu and C. Tellambura are with the Dept. of Electrical and Computer Engineering, University of Alberta, Edmonton, AB, Canada (e-mail: \{yuewu, chintha\}@ece.ualberta.ca).

T. Cui is with the Dept. of Electrical Engineering, California Institute of Technology, Pasadena, CA USA (e-mail: taocui@caltech.edu).

Digital Object Identifier 10.1109/LCOMM.2006.03016.
Assume the number of simultaneous users is not greater than the number of BS antennas. The bit stream of user $u$ is modulated and a block of $K$ modulated symbols is converted to a serial stream of $s_{u}[1], \cdots, s_{u}[K]$. This frequency-domain series is fed into a $K$-tap inverse fast Fourier transform (IFFT) to obtain the time-domain samples that are transmitted along with a cyclic prefix. The received signals at BS antenna $a$ are converted to a parallel stream and the cyclic prefix is removed. A $K$-tap fast Fourier transform (FFT) operator is used to obtain $y_{a}[1], \cdots, y_{a}[K]$, which are processed by the SDMA receiver to separate different users and provide the estimated data $\tilde{s}_{u}[1], \cdots, \tilde{s}_{u}[K]$ for user $u$.

The cyclic prefix is longer than the channel delay spread, and the $k$-th subcarrier output is given by [2]

$$
\mathbf{y}=\mathbf{H s}+\mathbf{n},
$$

where $\mathbf{y}=\left[y_{1}[k], \cdots, y_{A}[k]\right]^{T}$ is the received signals, $\mathbf{s}=$ $\left[s_{1}[k], \cdots, s_{U}[k]\right]^{T}$ are the transmitted user signals, $\mathbf{n}=$ $\left[n_{1}[k], \cdots, n_{A}[k]\right]^{T}$ is the additive white Gaussian noise, and

$$
\mathbf{H}=\left[\begin{array}{ccc}
h_{1,1}[k] & \cdots & h_{U, 1}[k] \\
\vdots & \ddots & \vdots \\
h_{1, A}[k] & \cdots & h_{U, A}[k]
\end{array}\right]
$$

is the frequency-domain channel transfer function matrix, where $h_{a, u}[k]$ is the channel gain between the $a$-th user and the $u$-th BS. We assume they are independent complex-Gaussian with zero mean and unit variance. The index $[k]$ is dropped for brevity.

\section{NEW DETECTOR}

The maximum likelihood (ML) detector is given by

$$
\tilde{\mathbf{s}}=\arg \min _{\mathbf{s} \in \mathcal{Q}^{U}}\|\mathbf{y}-\mathbf{H s}\|^{2},
$$

where $\mathbf{y} \in \mathcal{R}^{A}, \mathbf{H} \in \mathcal{R}^{A \times U}$ and $\mathcal{Q}=\{-(2 P-1), \cdots, 2 P+$ $1\}$ is a Pulse Amplitude Modulation (PAM) constellation of $P$ elements. Since a quadratue amplitude modulation (QAM) constellation can be decoupled into two real PAM constellations, the formulation (3) is also valid for QAM. $M$-ary phase shift keying ( $M$-PSK) will also be treated later. The brute-force ML detector must search all $P^{U}$ possible values of s. Hence, its complexity is exponential in $U$ (the number of variables to be estimated).

The original SD by Fincke and Phost [6] restricts the search space to the candidate signals within a hypersphere of an initial radius. Whenever a valid candidate is found, the radius may be updated. Eq. (3) is equivalent to

$$
\begin{aligned}
\tilde{\mathbf{s}} & =\arg \min _{\mathbf{s} \in \mathcal{Q}^{U}}\left(\mathbf{H}^{\dagger} \mathbf{y}-\mathbf{s}\right)^{H} \mathbf{R}^{H} \mathbf{R}\left(\mathbf{H}^{\dagger} \mathbf{y}-\mathbf{s}\right) \\
& =\arg \min _{\mathbf{s} \in \mathcal{Q}^{U}}(\mathbf{s}-\hat{\mathbf{s}})^{H} \mathbf{R}^{H} \mathbf{R}(\mathbf{s}-\hat{\mathbf{s}})
\end{aligned}
$$




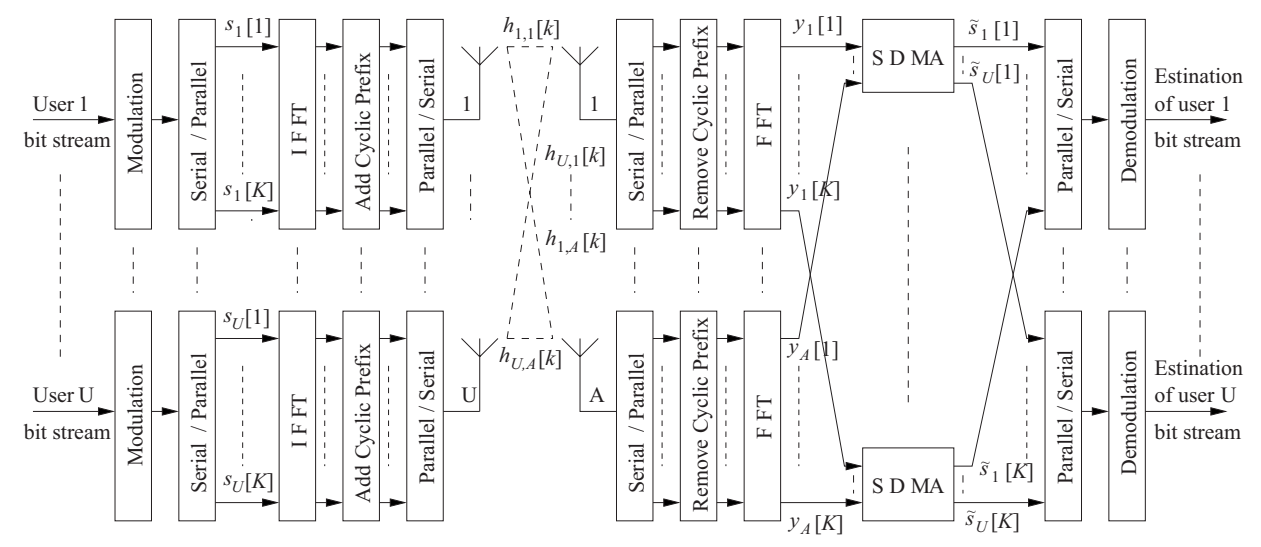

Fig. 1. System model.

where $\hat{\mathbf{s}}=\mathbf{H}^{\dagger} \mathbf{y}$ and $\mathbf{R}$ is an upper triangular matrix such that $\mathbf{R}^{H} \mathbf{R}=\mathbf{H}^{H} \mathbf{H}$. Let the entries of $\mathbf{R}$ be denoted by $r_{i j}, i \leq j$. Since the number of users $U$ is always less than or equal to the number of BS antennas $A, \mathbf{H}^{H} \mathbf{H}$ has full rank (positive definite). In the context of dispersive channels, the channel covariance matrix may still be singular or nearly singular, even though the number of users is less or equal to the number of BS antennas. This problem can be solved using the method in [8]. In this paper we assume the diagonal terms of $\mathbf{R}$ are non-zero $\left(r_{i i} \neq 0\right)$. A candidate is inside a hypersphere of radius $r$ when

$$
(\mathbf{s}-\hat{\mathbf{s}}){ }^{H} \mathbf{R}^{H} \mathbf{R}(\mathbf{s}-\hat{\mathbf{s}})=\sum_{i=1}^{U} r_{i i}^{2}\left\|s_{i}-\rho_{i}\right\|^{2} \leq r^{2}
$$

where $\rho_{i}=\hat{s}_{i}-\sum_{j=i+1}^{U} \frac{r_{i j}}{r_{i i}}\left(s_{j}-\hat{s}_{j}\right)$. We assume the initial radius $r$ is large enough so that the hypersphere (5) contains at least one candidate solution. The initial radius may be chosen according to noise variance [7].

Since each term in (5) is nonnegative, the partial sums must also be less than $r^{2}$. This fact can be used to derive a set of admissible values for $s_{u}(u=U, U-1, \ldots, 1)$ as

$$
\mathcal{L}_{u}=\left\{t \mid\left\lceil\rho_{u}-r_{u} / r_{u u}\right\rceil \leq t \leq\left\lfloor\rho_{u}+r_{u} / r_{u u}\right\rfloor, t \in \mathcal{Q}\right\}
$$

where $r_{u}^{2}=r^{2}-\sum_{i=u+1}^{U} r_{i i}^{2}\left\|s_{i}-\rho_{i}\right\|^{2}, s_{i} \in \mathcal{L}_{i},\lceil x\rceil$ is the smallest integer greater than or equal to $x$ and $\lfloor x\rfloor$ is the largest integer less than or equal to $x$. In (6), the signal constellation $\mathcal{Q}$ can be either PAM or QAM (expressed as two real PAM constellations). Eq. (6) generates all signal vectors satisfying (5), the number of which is much smaller than $|\mathcal{Q}|^{U}$.

If $\mathcal{Q}$ is $M$-PSK, (6) is not directly applicable ( $\mathcal{Q}$ is generally not decomposable into two real PAM constellations). We thus form the set of lists for $u=U, U-1, \ldots, 1$ as

$$
\mathcal{L}_{u}=\left\{t \mid r_{u u}^{2}\left\|t-\rho_{i}\right\|^{2} \leq r_{u}^{2}, t \in \mathcal{Q}\right\} .
$$

Once all the candidate signal vectors are generated via (6) or (7), the ML estimate is given by the candidate vector which minimizes (4). Putting all these ideas together, we get the following new detector algorithm:

1 Input $(\mathbf{y}, \mathbf{H}, r, \mathcal{Q})$;

$2[\mathbf{Q}, \mathbf{R}]=\operatorname{qr}(\mathbf{H}) ; \hat{\mathbf{s}}=\mathbf{H}^{\dagger} \mathbf{y} ; i=U ; l=1 ; \rho_{U}=\hat{s}_{U} ;$ $d^{2}=r^{2} ; r_{U}^{2}=r^{2} ; \mathcal{L}_{U}=\emptyset ;$ found $=0 ;$

\section{TABLE I}

\begin{tabular}{|c|c|}
\hline Parameter & Value \\
\hline Number of simultaneous users & 4 \\
Number of BS antennas & 4 \\
Bandwidth for each user (MHz) & 20 \\
Number of subcarriers & 64 \\
Number of data subcarriers & 48 \\
Cyclic Prefix Length & 16 \\
\hline
\end{tabular}

3 Generate $\mathcal{L}_{i}$ using (6) or (7);

4 If $\mathcal{L}_{i} \neq \emptyset$ and $i=1$, goto 8 ;

5 If $\mathcal{L}_{i} \neq \emptyset, \rho_{i-1}=\hat{s}_{i-1}-\sum_{j=i}^{U} \frac{r_{i-1, j}}{r_{i-1, i-1}}\left(s_{j}-\hat{s}_{j}\right)$, $r_{i-1}^{2}=r_{i}^{2}-r_{i i}^{2}\left\|s_{i}-\rho_{i}\right\|^{2}, i=i-1, l=1$, goto 3 ;

6 If $i=U$, if found $=1$, go to 9 , else increase $r, l=1$, goto 3 ;

$7 \quad i=i+1, l=l+1$, if $l>M$, goto 4 , else goto 3 ;

8 found $=1, \mathbf{s}^{*}=\mathbf{s}, \tilde{d}^{2}=r_{1}^{2}-r_{11}^{2}\left\|s_{1}-\rho_{1}\right\|^{2}, l=1$, goto 3 ;

9 If $\tilde{d}^{2}<d^{2}, d=\tilde{d}$, goto 7 , else return $\mathbf{s}^{*}$.

\section{CLS EXTENSION FOR NON-UNITARY SIGNALS}

An $M$-QAM vector can be represented as a weighted sum of $n / 2$ QPSK vectors when $M=2^{n}$ and $n$ is an even number. For example, 16QAM transmit vector $\mathrm{x}$ can be written as $\mathbf{s}=\sqrt{2} \mathbf{s}_{1}+\frac{\sqrt{2}}{2} \mathbf{s}_{2}$ where $\mathbf{s}_{1}$ and $\mathbf{s}_{2}$ are QPSK vectors. Thus (1) becomes

$$
\mathbf{y}=\left(\begin{array}{ll}
\sqrt{2} \mathbf{H} & \frac{\sqrt{2}}{2} \mathbf{H}
\end{array}\right)\left(\begin{array}{l}
\mathbf{s}_{1} \\
\mathbf{s}_{2}
\end{array}\right)+\mathbf{n} .
$$

A 16QAM system with $N$ transmit and $M$ receive antennas is equivalent to a QPSK system with $2 N$ transmit antenns and $M$ receive antennas. Similarly, any other $M$-QAM system can also be expressed as an equivalent QPSK system. Therefore, the CLS detector in [2] can be used with $M$-QAM.

\section{RESULTS AND CONCLUSION}

We consider a system compliant with the wireless local-area network (LAN) standard ETSI BRAN HIPERLAN/2 [9]. The system parameters are summarized in Table I.

We first compare the error performance of six detectors (the sensitive bits (SB) algorithm is from [5]) for QPSK (Fig. 2) and 16QAM (Fig. 3). In low SNR, the MMSE detector gains $2 \mathrm{~dB}$ over the V-BLAST detector [10], while the latter 


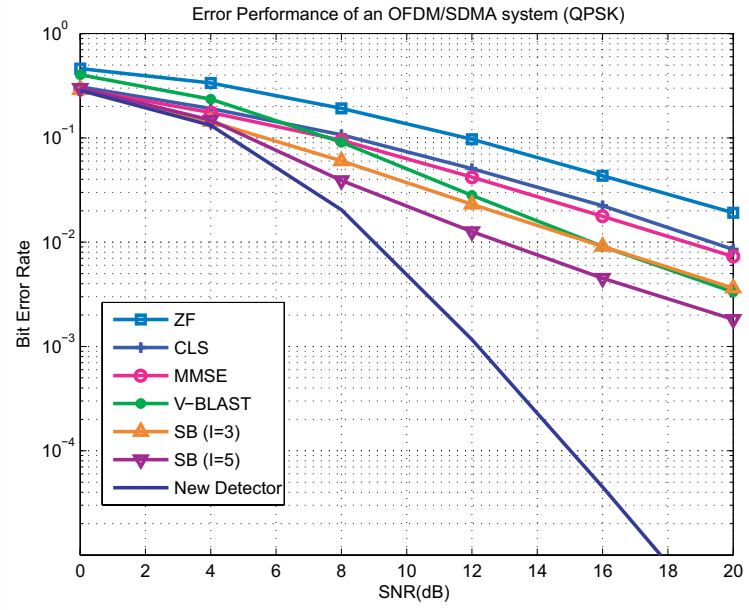

Fig. 2. Bit error rate of the detectors for QPSK.

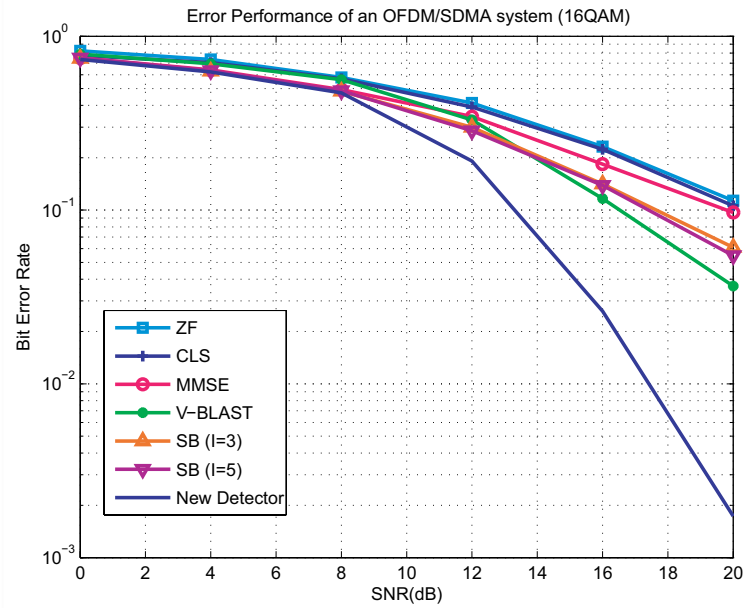

Fig. 3. Bit error rate of the detectors for 16QAM.

outperforms the former by more than $2 \mathrm{~dB}$ in high SNR. The MMSE detector performs slightly better than the CLS detector for both QPSK and 16QAM. The zero forcing (ZF) detector performs the worst in all SNR, and our new detector performs significantly better than the other five detectors. It gains about $7 \mathrm{~dB}$ over the V-BLAST detector at a BER of $10^{-2}$. This gain increases as the SNR increases. The diversity order is higher for our new detector than that for V-BLAST.

Fig. 4 shows the performance of the six detectors for the coded system. We use QPSK modulation and the industrystandard generator polynomials $g_{1}=133_{O C T}$ and $g_{2}=$ $171_{O C T}$ of rate $1 / 2$ convolutional code with constraint length 7. Our new detector significantly outperforms others.

Fig. 5 shows the computational complexity of the six detectors for QPSK. The complexity of the new detector is high for low SNR but decreases significantly as SNR increases, approaching that of the V-BLAST detector.

Using the sphere decoder, we proposed an optimal detector for SDMA/OFDM wireless systems. We also showed how to detect non-unitary signals with a constrained least squares (CLS) receiver. The results show that our new detector outperforms ZF, CLS, MMSE, V-BLAST, and SB detectors in both uncoded and coded systems.

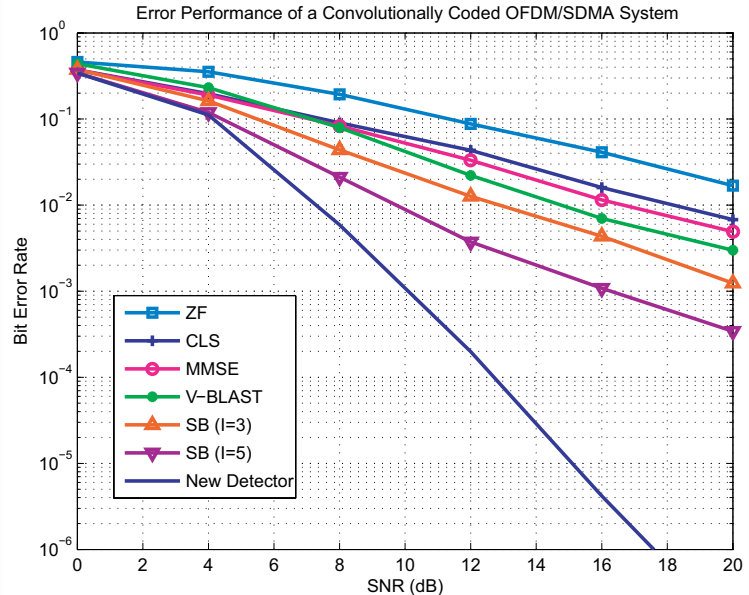

Fig. 4. Bit error rate with convolutional coding.

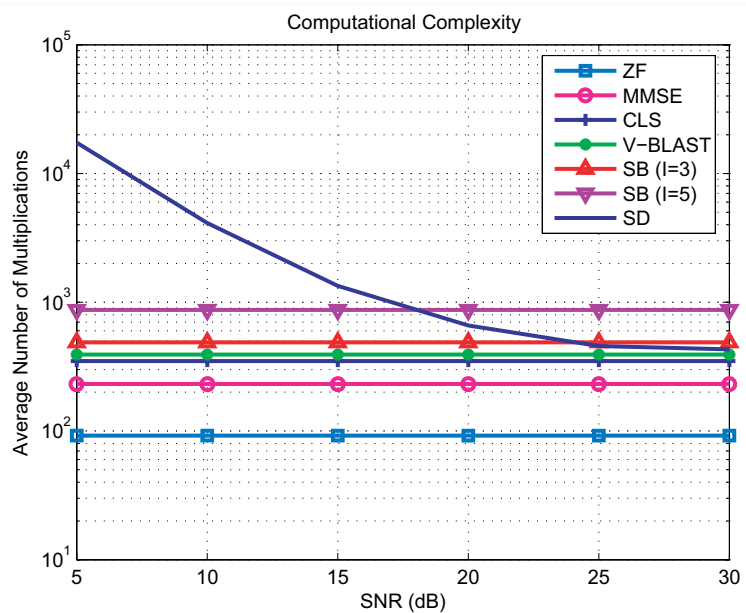

Fig. 5. Computational complexity of the detectors.

\section{REFERENCES}

[1] P. Vandenameele, L. Van der Perre, M. G. E. Engels, B. Gyselinckx, and H. J. De Man, "A combined OFDM/SDMA approach," IEEE J. Selected Areas Commun., vol. 18, pp. 2312-2321, Nov. 2000.

[2] S. Thoen, L. Deneire, L. Van der Perre, M. Engels, and H. De Man, "Constrained least square detector for OFDM/SDMA-based wireless networks," IEEE Trans. Wireless Commun., vol. 2, pp. 129-140, Jan. 2003.

[3] M. Y. Alias, A. K. Samingan, S. Chen, and L. Hanzo, "Multiple antenna aided OFDM employing minimum bit error rate multiuser detection," Electronics Lett., vol. 39, pp. 1769-1770, Nov. 2003.

[4] R. Sood and U. B. Desai, "Minimum probability of error demodulation for multipath OFDM-SDMA systems," in Proc. WCNC 04, vol. 2, pp. 948-953, Mar. 2004.

[5] J. Li, K. B. Letaief, R. S. Cheng, and Z. Cao, "Multi-stage low complexity maximum likelihood detection for OFDM/SDMA wireless LANs," in Proc. ICC 01, vol. 4, pp. 1152-1156, June 2001.

[6] U. Fincke and M. Pohst, "Improved methods for calculating vectors of short length in a lattice, including a complexity analysis," Mathematics of Computation, vol. 44, pp. 463-471, Apr. 1985.

[7] M. O. Damen, A. Chkeif, and J. C. Belfiore, "Lattice codes decoder for space-time codes," IEEE Commun. Lett., vol. 4, pp. 161-163, May 2000

[8] T. Cui and C. Tellambura, "An efficient generalized sphere decoder for rank-deficient mimo systems," IEEE Commun. Lett., vol. 9, pp. 423425, May 2005.

[9] ETSI TS 101475 V1.3.1 (2001-12), "Broadband radio access networks (BRAN); HIPERLAN Type 2; Physical (PHY) layer,” Dec. 2001.

[10] G. D. Golden, C. J. Foschini, R. A. Valenzuela, and P. W. Wolniansky, "Detection algorithm and initial laboratory results using V-BLAST space-time communication architecture," Electronics Lett., vol. 35, pp. 14-16, Jan. 1999. 